\title{
Role of Bicarbonate lon in the Postjunctional Action of Cocaine in the Smooth Muscle of the Rat Vas Deferens
}

\author{
Nagao SUZUKI and Yasuo GOMI \\ Department of Pharmacology, Faculty of Pharmaceutical Sciences, \\ Kanazawa University, Kanazawa 920. Japan
}

Accepted January 12, 1989

\begin{abstract}
In the HEPES-buffered physiological solution containing $20 \mathrm{mM}$ bicarbonate ion, cocaine not only increased the sensitivity to norepinephrine but enhanced the maximal contractions to norepinephrine and methacholine in the rat vas deferens. Cocaine also potentiated the high potassium-induced contractions. In the solution without bicarbonate ion, such effects of cocaine were lost except for the sensitivity increase to norepinephrine. These results suggest that bicarbonate ion is crucial for the postjunctional stimulatory action of cocaine in the rat vas deferens.
\end{abstract}

Cocaine has been reported to increase the sensitivity to norepinephrine in the vas deferens of rat and guinea pig (1-4). The dose-response curve to norepinephrine was shifted to the left by approximately one log unit by $10^{-6}-3 \times 10^{-5} \mathrm{M}$ cocaine. It is believed that increase in the sensitivity to norepinephrine in the vas deferens is due to the prejunctional action of cocaine to block the uptake of norepinephrine into the adrenergic nerve terminals as demonstrated in many adrenergically innervated smooth muscles (5). In addition, cocaine also enhanced the maximal responses to norepinephrine, acetylcholine and methacholine in the rat vas deferens $(1-3)$ and increased the sensitivity to acetylcholine slightly in the rat $(1,2)$ and consiaerably in the guinea pig vas deferens (4). These stimulatory effects of cocaine have been suggested to be due to the postjunctional action of cocaine to affect the properties of smooth muscle cells of the vas deferens (1,4). Although the definite mechanism for this action of cocaine has not been elucidated. Araki et a!. (4) suggested that cocaine facilitates the influx of calcium in the guinea pig vas deferens.

So far, effects of cocaine have been studied in Krebs' or Tyrode's solution containing high concentrations of bicarbonate from 10 to $25 \mathrm{mM}$. Since the role of bicar- bonate in the action of cocaine has not been heeded, we examined the effects of cocaine in HEPES ( $\mathrm{N}$-2-hydroxyethylpiperazine- $\mathrm{N}$ : 2-ethanesulfonic acid)-buffered solution with or without bicarbonate ion. The results indicate that bicarbonate is requisite for the postjunctional, but not for the prejunctional. action of cocaine in the rat vas deferens.

A pair of vasa deferentia from male Wistar rats $(250-350 \mathrm{~g})$ were placed in a petri dish containing HEPES-buffered physiological solution with or without $20 \mathrm{mM} \mathrm{NaHCO}_{3}$. The bicarbonate-containing solution had the following composition: $120 \mathrm{mM} \mathrm{NaCl}, 6 \mathrm{mM}$ $\mathrm{KCl}, 2 \mathrm{mM} \mathrm{CaCl}_{2}, 1 \mathrm{mM} \mathrm{MgCl}, 1 \mathrm{mM}$ $\mathrm{NaH}_{2} \mathrm{PO}_{4}, 20 \mathrm{mM} \mathrm{NaHCO}, 5 \mathrm{mM}$ HEPES and $5.5 \mathrm{mM}$ glucose: the $\mathrm{pH}$ was adjusted to 7.3 at $36^{\circ} \mathrm{C}$ by $1 \mathrm{~N} \mathrm{HCl}$. Bicarbonate-free solution contained $140 \mathrm{mM} \mathrm{NaCl}$ instead of $120 \mathrm{mM} \mathrm{NaCl}$, and the other components were the same as those in the bicarbonatecontaining solution: the $\mathrm{pH}$ was adjusted to 7.3 by $1 \mathrm{~N} \mathrm{NaOH}$. After being cleaned of adhering fat and blood vessels, the epididymal portion of the vas deferens was mounted in an organ chamber $(10 \mathrm{ml})$ with a resting tension of $0.5 \mathrm{~g}$ and allowed to equilibrate for $60 \mathrm{~min}$ in either solution. The bathing solution in the chamber was continuously exchanged with fresh solution warmed to $36^{\circ} \mathrm{C}$ flowing at a rate of $3 \mathrm{ml} / \mathrm{min}$ and was bubbled with pure 

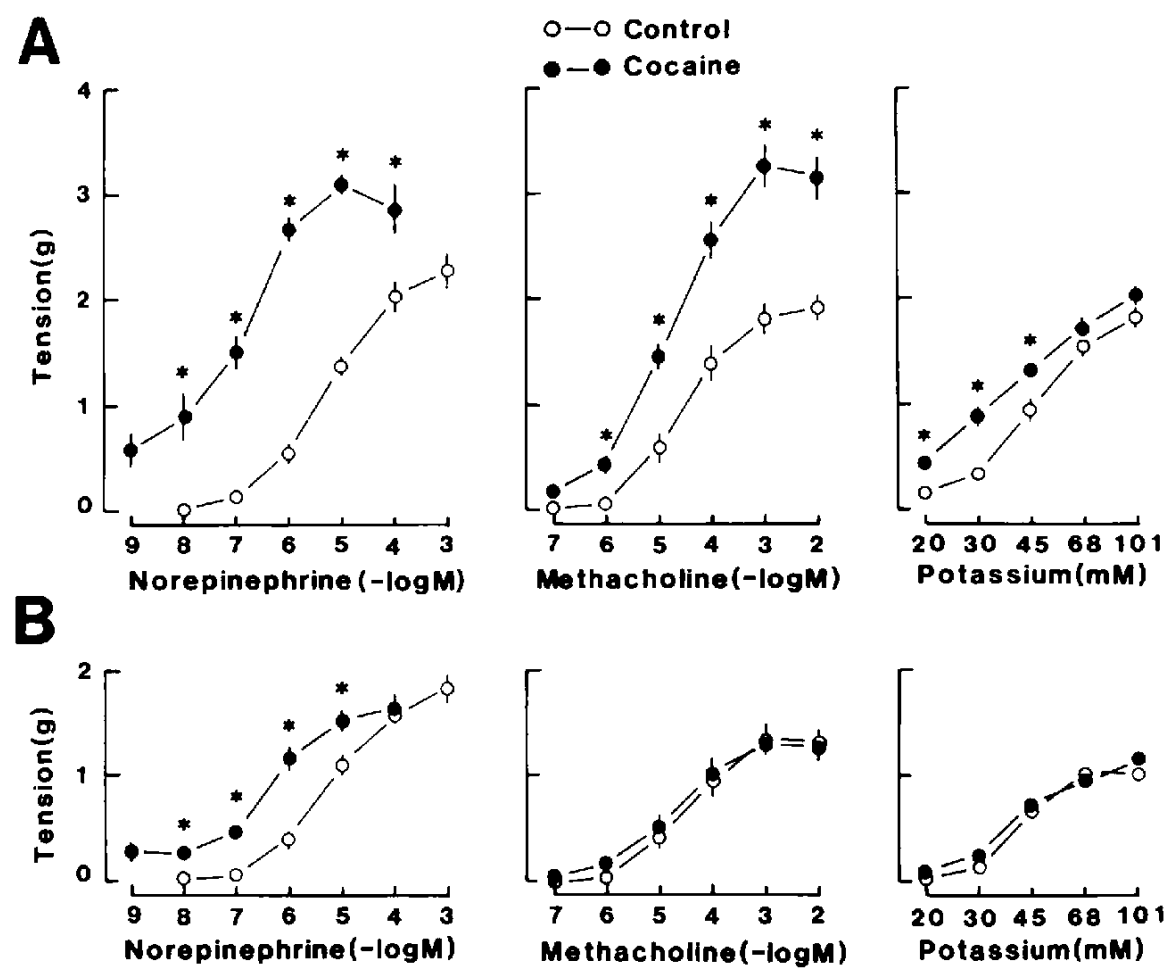

Fig. 1. Effects of $10^{-5} \mathrm{M}$ cocaine on the dose-response curves to norepinephrine, methacholine and potassium in HEPES-buffered physiological solution with (A) or without (B) $20 \mathrm{mM}$ bicarbonate ion. Asterisks indicate that the values are statistically different from the control at $P=0.05$. When analyzed with Student's unpaired t-test. The number of preparations is 6 for all dose-response curves. Vertical bars represent standard errors of the mean.

oxygen. The dose-response relationship was determined by non-cumulative application of agonists. Increasing concentrations of agonists were applied with an interval of $15 \mathrm{~min}$ between the applications, and the peak amplitudes of the contractions induced by each concentration were used to construct the dose-response curve. After control doseresponse curves were determined in the absence of cocaine, the preparations were allowed to rest for $30 \mathrm{~min}$, and then the doseresponse curves in the presence of $10^{-5} \mathrm{M}$ cocaine were obtained. Cocaine was added to the organ chamber $10 \mathrm{~min}$ prior to the application of each concentration of agonist. For the dose-response curve to potassium, preparations were pretreated with $10^{-6} \mathrm{M}$ dibenamine for 10 min during the equilibration period. High potassium solutions were made by replacing $\mathrm{NaCl}$ in physiological solution with equimolar $\mathrm{KCl}$.

Figure 1 shows the effects of $10^{-5} \mathrm{M}$ cocaine on the dose-response curves to norepinephrine, methacholine and potassium in the HEPES-buffered solution with or without $20 \mathrm{mM}$ bicarbonate ion. In the presence of bicarbonate ion (Fig. 1A), cocaine shifted the dose-response curve to norepinephrine to the left by 1.6 log units expressed as change in ED50 values and enhanced the maximal response by $41 \%$. Cocaine also enhanced the maximal response to methacholine by $69 \%$ in the bicarbonatecontaining solution. Sensitivity to methacholine was almost unchanged by cocaine. Cocaine potentiated the contractions to potassium at up to $45 \mathrm{mM}$. Maximal contractions to potassium were slightly increased by cocaine, although the increment was not statistically significant. 
In the absence of bicarbonate ion (Fig. 1B), the contractions to norepinephrine and methacholine were slightly smaller than those in the bicarbonate-containing solution. Contractions to high potassium were greatly reduced in the bicarbonate-free solution; the maximal responses were $60.3 \pm 4.5 \%$ (mean \pm standard errors, $n=6$ ) of those in the bicarbonate-containing solution. Al though cocaine still shifted the dose-response to norepinephrine to the left by $1.2 \mathrm{log}$ units in the bicarbonate-free solution, the maximal response was not enhanced by cocaine. Furthermore, neither the enhancement of the maximal response to methacholine nor the potentiation of potassium-induced contractions was seen in the bicarbonatedepleted solution.

Figure 2 shows the effects of supplementing bicarbonate ion on the enhancing action of cocaine on the maximal responses to methacholine. In this experiment, cocaine

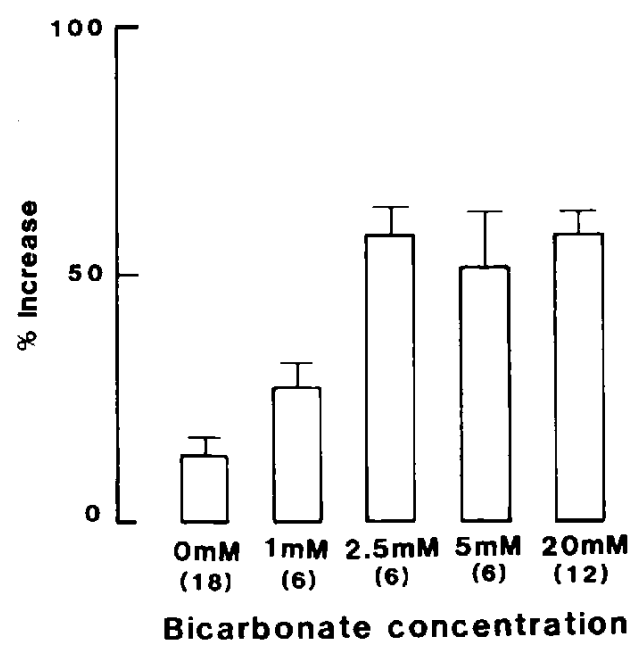

Fig. 2. Effects of various concentrations of bicarbonate in the HEPES-solution on the enhancing action of cocaine for the maximal response to methacholine. Ordinate, \% increase in the response to $10^{-3} \mathrm{M}$ methacholine by $10^{-5} \mathrm{M}$ cocaine. Abscissa. concentration of bicarbonate ion supplemented to the bicarbonate-free HEPES solution. After the effects of cocaine in bicarbonate-free solution were determined, the solution was exchanged to a bicarbonate-containing one, and the effects of cocaine were again determined. The numbers in the parentheses are the numbers of preparations used. Vertical bars represent standard errors of the mean. slightly increased the response to $10^{-3} \mathrm{M}$ methacholine in the bicarbonate-free solution. Addition of as low as $1 \mathrm{mM}$ bicarbonate considerably reinforced the enhancing action of cocaine. Therefore, it seems possible that the small increment by cocaine in the bicarbonate-free solution is due to residual bicarbonate in the extracellular space of the vas deferens. Full recovery of the action of cocaine was obtained at $2.5 \mathrm{mM}$ bicarbonate ion.

The present results clearly demonstrated that postjunctional effects of cocaine in the rat vas deferens were dependent on the presence of bicarbonate ion, at least, in the HEPES-buffered solution. Prejunctional effects of cocaine seem to be independent of bicarbonate ion since the extent of sensitivity increase to norepinephrine by cocaine was similar in the bicarbonate-containing and bicarbonate-free solution.

Bicarbonate ion could be involved in various functions of the smooth muscle. It is most likely that bicarbonate ion is responsible for the regulation of extracellular and intracellular $\mathrm{pH}$ under physiological conditions. In the present study, extracellular $\mathrm{pH}$ was maintained at 7.3 in the bathing solutions regardless of the presence or absence of bicarbonate ion. However, intracellular $\mathrm{pH}$ may be different between the two solutions. If the sites of action for the postjunctional effects of cocaine were inside of the smooth muscle cells and only the cationic or nonionic form of cocaine was the active substance, variation of intracellular $\mathrm{pH}$ mav influence the effects of cocaine.

It has been reported that bicarbonate ion exchanges with chloride ion in the guinea pig vas deferens, and thus bicarbonate ion was suggested to be responsible for the regulation of intracellular chloride concentration (6). Perturbation of chloride metabolism by deprivation of bicarbonate ion may affect the membrane potential or the transport of other ions, resulting in loss of cocaine action.

Specific binding of $\left[{ }^{3} \mathrm{H}\right]$ cocaine to the membrane fraction has recently been shown in peripheral organs of rats including smooth muscles such as the ileum (7). although the function of these binding sites has not been 
clarified. In liver membrane, which showed the highest concentration and affinity of cocaine binding, no measurable uptake of $\left[{ }^{3} \mathrm{H}\right]$ norepinephrine was observed (7). Despite the obscure function, existence of binding sites for cocaine in various peripheral organs may imply that there are also specific binding sites for cocaine in the smooth muscle of vas deferens and that the interaction of cocaine with such sites is the initial event for exertion of the postjunctional effects of cocaine. Bicarbonate ion may be necessary for cocaine to bind to these specific sites in the smooth muscle of rat vas deferens.

Finally, bicarbonate may affect the voltagedependent calcium channels in the rat vas deferens, since the contractions in response to potassium were significantly decreased in the bicarbonate-free solution. As discussed above, the change in membrane potential may be partly responsible for the reduction in the response to lower concentrations of potassium. However, this explanation may not apply to the reduced response to the maximal concentration of potassium, which would produce nearly full depolarization, thereby making the difference in the membrane potential negligible. Thus, we suppose that the functions of voltage-dependent calcium channels in the rat vas deferens are impaired under the bicarbonate-deprived condition. The possibility that cocaine enhances the contractile responses by affecting the bicar- bonate-dependent characteristics of voltagedependent calcium channels is under investigation.

\section{References}

1 Kasuya, Y. and Goto, K.: The mechanism of supersensitivity to norepinephrine induced by cocaine in rat isolated vas deferens. Eur. J. Phar macol. 4, 355-362 (1968)

2 Kasuya, Y. and Suzuki, N.: Regional differences in the effects of denervation, cocaine and chronic reserpine administration on the responses of the rat vas deferens to norepinephrine and acetylcholine. Arch. Int. Pharmacodyn. Ther. 236, 202-213 (1978)

3 Bradley, L., Doggrell, S.A. and Edmonds, S.C.: Cocaine potentiates the responses to methacholine and noradrenaline in the rat vas deferens. J. Pharm. Pharmacol. 37, 369-372 (1985)

4 Araki, K., Muramatsu, M. and Gomi, Y.: Pharmacological studies on supersensitization. VI. Effect of cocaine on the utilization of calcium in acetylcholine-induced contraction of isolated vas deferens of guinea pig. J. Pharmacobiodyn. 5 , 693-698 (1982)

5 Trendelenburg, $\mathrm{U} .:$ Mechanisms of supersensitivity and subsensitivity to sympathomimetic amines. Pharmacol. Rev. 18, 629-640 (1966)

6 Aickin, C.C. and Brading, A.F.: The role of chloride-bicarbonate exchange in the regulation of intracellular chloride in guinea-pig vas deferens. J. Physial. (Lond.) 349, 587-606 (1984)

7 Calligaro, D.O. and Eldefrawi, M.E.: Central and peripheral cocaine receptors. J. Pharmacol. Exp. Ther. 243, 61-68 (1987) 